23

\title{
Исследование рефрактометром дифференциального типа влияния оптической плотности текущей жидкости на погрешность измерения показателя преломления
}

\author{
(C) В.В. Давыдов, А.В. Мороз \\ Санкт-Петербургский политехнический университет Петра Великого, \\ 195251 Санкт-Петербург, Россия \\ e-mail: Davydov_vadim66@mail.ru
}

Поступила в редакцию 08.04.2020 г.

В окончательной редакции 08.04.2020 г.

Принята к публикации 20.05.2020 г.

\begin{abstract}
Установлены факторы, которые оказывают влияние на погрешность измерения показателя преломления $n$ в текущей жидкости (изменение ее оптической плотности и температуры). При использовании для измерений $n$ текущей жидкости проточного рефрактометра дифференциального типа на погрешность измерения показателя преломления дополнительное влияние оказывает рассеяние излучения в кюветном преобразователе и клиновидность стекол кюветы. Разработана новая конструкция дифференциального рефрактометра для исследования влияния оптической плотности текущей жидкости на погрешность измерения $n$. Получены новые результаты, подтверждающие влияние изменения оптической плотности текущей жидкости на погрешность измерения показателя преломления $n$. Предложена методика компенсации влияния изменения оптической плотности на погрешность измерения $n$.
\end{abstract}

Ключевые слова: показатель преломления, текущая жидкость, рефрактометр дифференциального типа, погрешность измерения.

DOI: $10.21883 /$ OS.2020.09.49869.133-20

\section{Введение}

Одной из наиболее сложных задач, которые решает спектроскопия, является контроль состояния жидких сред, протекающих в трубопроводах различного диаметра [1-6]. Особенно если проводимые исследования не должны вносить необратимые изменения в ее физическую структуру и химический состав. Поэтому при их проведении предпочтение отдается бесконтактным методам [5-9]. Среди них наибольшими преимуществами обладают методы, основанные на использовании явления рефракции [10-14] и ядерного магнитного резонанса (ЯМР) [15-19]. Метод рефракции в отличие от ЯМР, кроме обеспечения стерильности измерительного процесса, позволяет одним прибором контролировать состояние жидкости, находящейся как в стационарном, так и в текущем состоянии. Основным параметром, по которому осуществляется контроль состояния среды, является показатель преломления $n$.

В настоящее время для решения новых задач при проведении научных исследований, для определения наличия примесей в среде или контроля состояния технологического процесса, например, на фармакологическом производстве необходимо обеспечить измерения значений $n$ с погрешностью $10^{-4}$ и меньше [20-23]. В эту величину погрешности входят все негативные факторы и возможные ошибки измерений, которые возникают по различным причинам. Наиболее сложной проблемой, которую необходимо решить для обеспечения этих зна- чений погрешностей измерения $n$, является уменьшение влияния оптической плотности в текущей жидкости на результат определения $n$. В рефрактометрах, в которых жидкость при измерении $n$ находится в стационарном состоянии, для решения этой задачи используются различные компенсационные схемы. Это позволяет сделать несущественным влияние изменения оптической плотности в стационарной жидкости на погрешность измерения $n$.

При исследовании текущей жидкости использование компенсационных устройств возможно только, если рефрактометр построен по дифференциальной схеме [20-25]. В зависимости от типов конструкции проточных рефрактометров дифференциального типа влияние различных погрешностей на результат измерения $n$ то возрастает, то уменьшается. Разделить вклад оптической плотности в погрешность измерения $n$ от вкладов других явлений и факторов при использовании разработанных ранее конструкций рефрактометров дифференциального типа невозможно. Исследовать влияние оптической плотности на погрешность измерения $n$ одновременно с другими погрешностями, влияние которых на результат измерения $n$ не скомпенсировано, не имеет смысла.

С другой стороны, без компенсации погрешностей, связанных с оптической плотностью, получить значение погрешности $n$ менее $10^{-4}$ крайне сложно.

Поэтому нами в работе последовательно решаются несколько задач. Разработка новой конструкции проточного рефрактометра дифференциального типа, в кото- 
рой влияние погрешностей, не связанных с оптической плотностью, на результат измерения $n$ был бы несуществен. Реализация в разработанной конструкции рефрактометра (с учетом ее особенностей) нового метода измерения $n$ текущей жидкости. Исследование влияние изменения оптической плотности текущей жидкости на погрешность измерения $n$.

\section{Экспериментальная установка и методика исследования влияния изменения оптической плотности на погрешность измерения $n$}

В разработанных ранее конструкциях проточных рефрактометров дифференциального типа для измерения показателя преломления $n$ текущей среды используется метод разностной призмы Андерсона с дифференциальной кюветой $[11,12,20,22,24,25]$. Базовая конструкция дифференциальной (разностной) кюветы состоит из двух или трех емкостей. По одной емкости (проточная кювета) движется исследуемая жидкость. Ее показатель преломления $n_{m}$ необходимо измерять. Во второй емкости (замкнутая кювета) или двух емкостях находится жидкая среда с известным показателем преломления $n$. Замкнутая кювета размещается внутри проточной или проточная внутри двух замкнутых для выравнивания температур в двух средах [22,24,25].

Измерение показателя преломления $n_{m}$ в конструкции проточного рефрактометра дифференциального типа основано на регистрации угла отклонения лазерного излучения, выходящего из разностной кюветы, которое пропорционально $\Delta n=n_{m}-n_{c}$. На основе этой зависимости определяется формативный параметр дифференциального рефрактометра $\beta[22,24,25]$ :

$$
\beta=\Delta n \operatorname{tg}(\gamma),
$$

где $\gamma$ - угол между входящим лучом и перпендикуляром к границе раздела проточной и замкнутой емкостей.

Угол $\gamma$ определяется положением оси оптического клина (компенсатора), вращая который достигается максимальное значение отношения сигнал/шум оптического сигнала, регистрируемого фотоприемником [10,20,24]. Проведенные нами исследования показали, что при таком построении конструкции дифференциального рефрактометра на точность измерения $n_{m}$ влияют следующие погрешности.

1. Погрешность $\delta_{1}(\Delta n)$, связанная с напряжением разбаланса на фотоэлектрическом преобразователе. Для его компенсации используется оптический клин, который вносит дополнительную погрешность за счет увеличения числа отражений между оптическими элементами лазерного излучения.

2. Динамическая погрешность, связанная с изменением температуры текущей жидкости. Эта погрешность существенно влияет на результат измерения при скачкооб- разном изменении температуры или расхода $q$ текущей жидкости.

3. Погрешностью $\delta_{2}(\Delta n)$, связанной с наличием в рефрактометрах дифференциального типа на основе кюветы Андерсона транспортного звена для отбора текущей жидкости. Наличие этого звена приводит к появлению времени запаздывания $\tau_{d}$ при проведении измерений.

4. Погрешность $\delta(\Delta n)$, связанная с изменением оптической плотности текущей жидкости по сечению трубопровода.

Анализ полученных нами результатов экспериментальных исследований, в которых были установлены зависимости для описания динамической погрешности и погрешностей транспортного звена, а также использование соотношений для других погрешностей, полученных в работах [12-14,20-22,24,25], позволил получить для рефрактометров дифференциального типа формулу для определения погрешности измерения $\delta n_{m}$ :

$$
\begin{aligned}
& \delta n_{m}=\delta(\Delta n)+\delta_{1}(\Delta n)+\delta_{2}(\Delta n)+b\left(T_{f}-T_{c}\right) \\
& +\left(b\left(\tau_{c} \tau_{f t} p_{1}+k_{1} \tau_{f t}+\tau_{c}\right) L^{p_{1}}\right) /\left(\left(p_{1}-p_{2}\right)\left(p_{1}-p_{3}\right)\right) \\
& -\left(b\left(\tau_{c} \tau_{f t} p_{2}+k_{2} \tau_{f t}+\tau_{c}\right) L^{p_{2}}\right) /\left(\left(p_{1}-p_{2}\right)\left(p_{2}-p_{3}\right)\right) \\
& +\left(b\left(\tau_{c} \tau_{f t} p_{3}+k_{2} \tau_{f t}+\tau_{c}\right) L^{p_{3}}\right) /\left(\left(p_{1}-p_{3}\right)\left(p_{2}-p_{3}\right)\right),
\end{aligned}
$$

где $p_{1}=-1 / \tau_{d}$

$p_{2,3}=-\left(\left(\tau_{c}-\tau_{f t}\right) \pm \sqrt{\left(\tau_{c}-\tau_{t f}\right)^{2}-4 k_{1} \tau_{c} \tau_{t f}}\right) /\left(2 \tau_{c} \tau_{f t}\right)$,

$\tau_{c}$ - постоянная времени замкнутой кюветы, $\tau_{t f}-$ постоянная времени проточной кюветы, $L-$ расстояние от дифференциальной кюветы до фотоприемника, $b$ - температурный градиент показателя преломления текущей жидкости, $k_{1}$ и $k_{2}-$ коэффициенты, характеризующие размеры проточной и замкнутой кювет, массу и теплоемкость жидкостей, в них находящихся, и прочие; $T_{f}$ - температура проточной жидкости; $T_{c}-$ температура эталонной жидкости.

Анализ соотношения (2) показывает, что проводить исследования влияния оптической плотности на погрешность измерения $\delta n_{m}$ в разработанных в настоящее время конструкциях проточных рефрактометров дифференциального типа некорректно. Так как уменьшая влияние на точность измерения одной погрешности, например, увеличивая расход жидкости (в этом случае уменьшается время запаздывания $\tau_{d}$, на транспортном участке, но возрастает вероятность образования застойных зон), увеличивается вклад от других погрешностей измерения $n_{m}$. При увеличении расхода жидкости $q$ возрастают погрешности, связанные с изменением температуры и оптической плотности. Кроме того, в дифференциальной кювете есть повороты и углы. Эти факторы также увеличивают влияние различных погрешностей при увеличении $q$. Поэтому нами для исследования влияния оптической плотности на погрешность измерения $\delta n_{m}$ была разработана новая конструкция экспериментальной 
установки (лабораторный макет рефрактометра дифференциального типа). Ее структурная схема представлена на рис. 1.

Излучение от полупроводникового лазера 1 с $\lambda=632.8 \mathrm{~nm}$ поступает на оптическую систему 3 (призменный коллиматор), на выходе которого формируется параллельный пучок заданной формы. В лабораторном макете рефрактометра используются кюветы цилиндрической формы: проточная 6 и замкнутая 5. Текущая жидкость движется по проточной кювете 6 в направлении, перпендикулярном относительно поступающего на ее стенку лазерного излучения.

Кюветы 5 и 6 расположены в вертикальном положении. Этот новый способ расположения кювет позволяет исключить наличие пустот в проточной кювете при малых расходах жидкости в отличие от ранее используемых конструкций кювет в дифференциальных рефрактометрах. Такое размещение кювет позволяет полностью исключить образование в них застойных зон и обеспечить более эффективный теплообмен между текущей жидкостью и эталонной по сравнению с ранее используемыми конструкциями рефрактометров. Это существенно снижает влияние вклада погрешности от изменения температуры на погрешности измерения $n_{m}$. Такое расположение кювет 5 и 6 позволяет исключить из (2) слагаемые, связанные с погрешностями от времени запаздывания и напряжения разбаланса, а также функциональный член $\delta_{2}(\Delta n)$, так как лазерное излучение поступает на стенку трубопровода под прямым углом. В этом случае соотношение (2) для определения погрешности измерения $n_{m}$ приобретает следующий вид:

$$
\delta(\Delta n)=0.993 \frac{\alpha^{2} \Delta d}{L \Delta n},
$$

где $\Delta d=d_{m}-d_{0}-$ изменение оптической плотности исследуемой жидкости $d_{m}$ относительно начального значения $d_{0}, a-$ полуширина светового пучка, $L-$ расстояние от дифференциальной кюветы до фотодиодной линейки.

С учетом особенностей размещения кювет и воздействия на текущую и эталонную жидкость лазерного излучения нами был разработан новый метод измерения показателя преломления $n_{m}$ с использованием дифференциальной схемы регистрации оптического сигнала. Для его реализации в конструкцию рефрактометра была включена призма Дове 4 (рис. 1). На выход призмы Дове 4 поступает половина лазерного излучения после оптической системы 3. На выходе из призмы Дове излучение изменяет направление на противоположное первоначальному. Далее все лазерное излучение поступает на замкнутую кювету 5, потом проходит через проточную кювету 6 с исследуемой жидкостью и через кювету 5 поступает на делительную призму 7. На делительной призме 7 происходит разделение световых потоков. В результате одна часть светового потока уменьшает амплитуду сигнала первой фотодиодной линейки и увеличивает освещенность второй. Другая часть светового потока увеличивает амплитуду сигнала первой фотодиодной линейки и уменьшает второй фотодиодной линейки. Установлено, что величина разности амплитуд нормированных выходных сигналов с фотодиодных линеек пропорциональна значениям $\beta$ и $L$ (расстояние от стенки кюветы 6 до фотодиодной линейки). В этом случае формула (1) для определения формативного параметра $\beta$ приобретает следующий вид:

$$
\beta=A_{a r}^{1}-A_{a r}^{2}=L K_{a r} \Delta n=L K_{a r}\left(n_{m}-n_{c}\right),
$$

где $K_{a r}$ - коэффициент пропорциональности, $A_{a r}^{1}, A_{a r}^{2}-$ нормированные выходные сигналы с фотодиодных линеек 1 и 2.

Нормировка выходного сигнала $A$ с фотодиодной линейки осуществляется по следующему принципу:

$$
A=\frac{\sum_{i=1}^{512} A_{i}}{A_{\max }},
$$

где $A_{\max }$ - максимальное значение сигнала освещенности с элемента фотодиодной линейки, $A_{i}-$ амплитуда сигнала с элемента фотодиодной ячейки, $i-$ номер элемента.

Коэффициент пропорциональности $K_{a r}$ определяется при предварительной градуировке рефрактометра. Для этого используются две жидкости с известными показателями преломления: дистиллированная вода и этанол (этиловый спирт). В результате экспериментов установлено, что для прозрачных сред значение $K_{a r}$ не изменяется. Температура текущей жидкости в проточной кювете и эталонной жидкости в замкнутой кювете контролируется датчиками температуры 12. Информация от них через специальные устройства 13 поступает на устройство обработки информации 10.

Для регистрации излучения в разработанном нами в лабораторном макете рефрактометра используются две фотодиодные линейки TSL1406RS, состоящие из 512 фоточувствительных сенсоров (компания AMS-TAOS USA), с длиной фоточувствительного слоя $40.16 \mathrm{~mm}$. В конструкции фотодиодной линейки TSL1406RS перед фоточувствительным слоем нет фокусирующих оптических элементов, как в других моделях линеек. Поэтому влияние эффектов, связанных с неоднократным отражением лазерного излучения между фоточувствительным слоем и гранями призмы 7, на отношение сигнал/шум на фоточувствительных сенсорах несущественно.

С фотодиодных линеек 8 сигналы через аналогово-цифровые преобразователи поступают в устройство обработки информации, разработанное на основе микроконтроллера STM32 (ARM Cortex M3 core STM32F100RBT6B). C микроконтроллера информация поступает на ноутбук, который используется для определения значения $n_{m}$ и визуализации различной информации. 


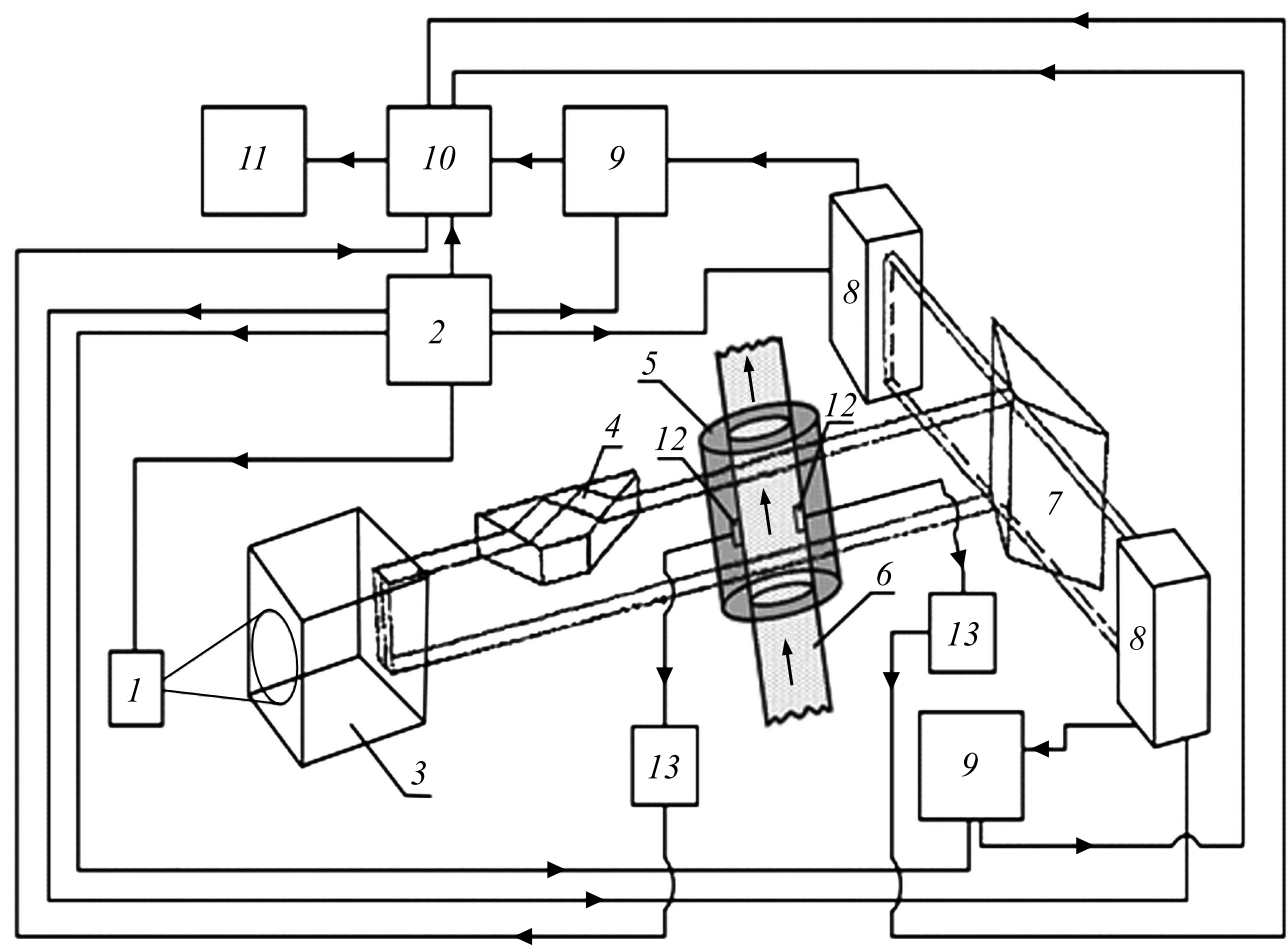

Рис. 1. Структурная схема экспериментальной установки: 1 - полупроводниковый лазер; 2 - многофункциональный источник питания; 3 - оптическая система; 4 - призма Дове; 5 - замкнутая кювета; 6 - проточная кювета; 7 - делительная призма; 8 - фотодиодная линейка; $9-$ АЦП; $10-$ устройство обработки и управления; 11 - ноутбук; 12 - датчик температуры; $13-$ устройство для обработки информации с датчика температуры.

\section{Результаты экспериментальных исследований и их обсуждение}

Надежность и достоверность процесса измерения показателя преломления $n_{m}$ текущей жидкости с использованием новой разработанной конструкцией рефрактометра (лабораторный макет) была проверена на нескольких жидких средах для различных температур $T_{f}$. На рис. 2 в качестве примера представлены зависимости изменения показателя преломления текущих жидкостей от температуры.

Показатели преломления этих жидких сред были измерены с помощью промышленного проточного рефрактометра PRM-100 alpha (company ATAGO). Полученные результаты показателей преломления совпали в пределах погрешности измерений. Кроме того, значения показателей преломления, измеренных на разработанном лабораторном макете рефрактометре дифференциального типа, совпали со значениями показателей преломления, полученными ранее с использованием других конструкций рефрактометров [12-14,20-22,24-26].

На рис. 3 в качестве примера представлена зависимость показателя преломления $n_{m}$ текущей дистиллированной воды от ее солености при различных температуpax.

Данная зависимость была исследована с использованием проточного рефрактометра PRM-100 (company

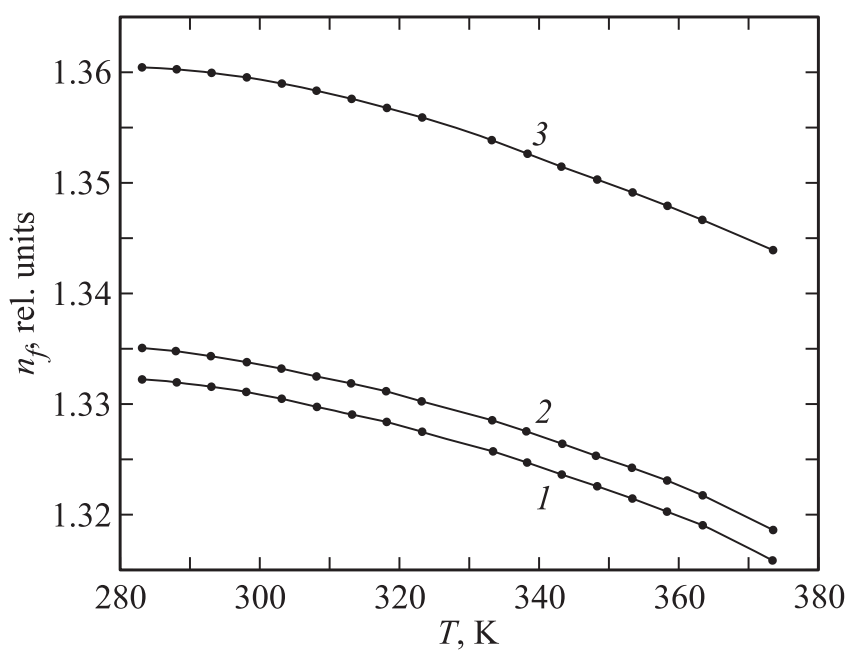

Рис. 2. Зависимость показателя преломления $n_{m}$ от температуры $T_{f}$. Графикам 1,2,3 соответствуют следующие среды: дистиллированная вода, водопроводная вода, этиловый спирт.

ATAGO). Полученные значения показателей преломления совпали в пределах погрешности измерений. Значения показателей преломления при различной температуре среды с различной соленостью, измеренные разработанным лабораторным макетом рефрактометра, совпали в пределах погрешности измерения с результатами, по- 


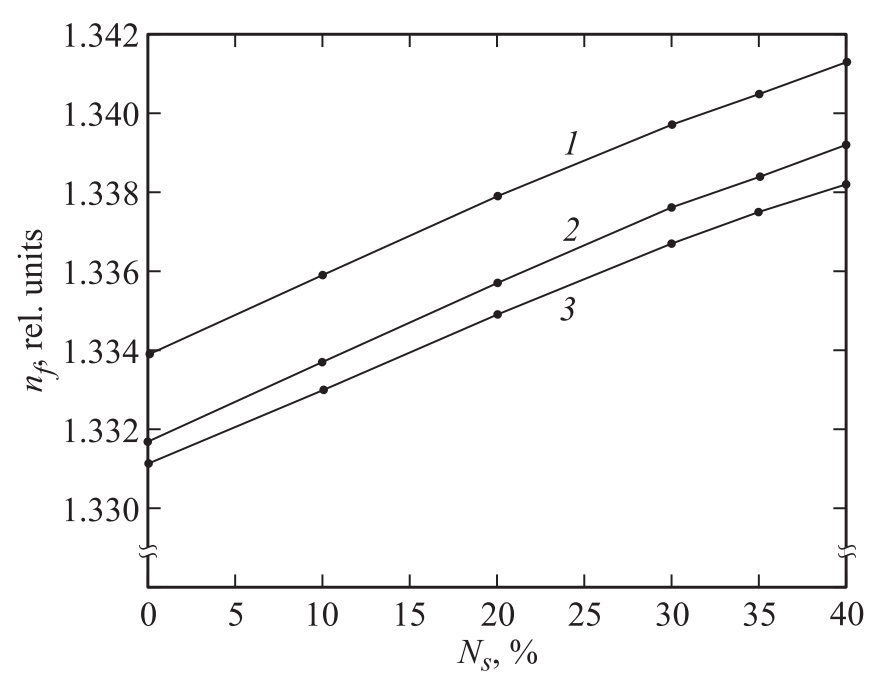

Рис. 3. Зависимость показателя преломления $n_{m}$ от солености воды $N_{s}$ для различных температур $T_{f}$. Графикам 1, 2, 3 соответствует $T_{f}=283.1,293.1$ и $303.2 \mathrm{~K}$.

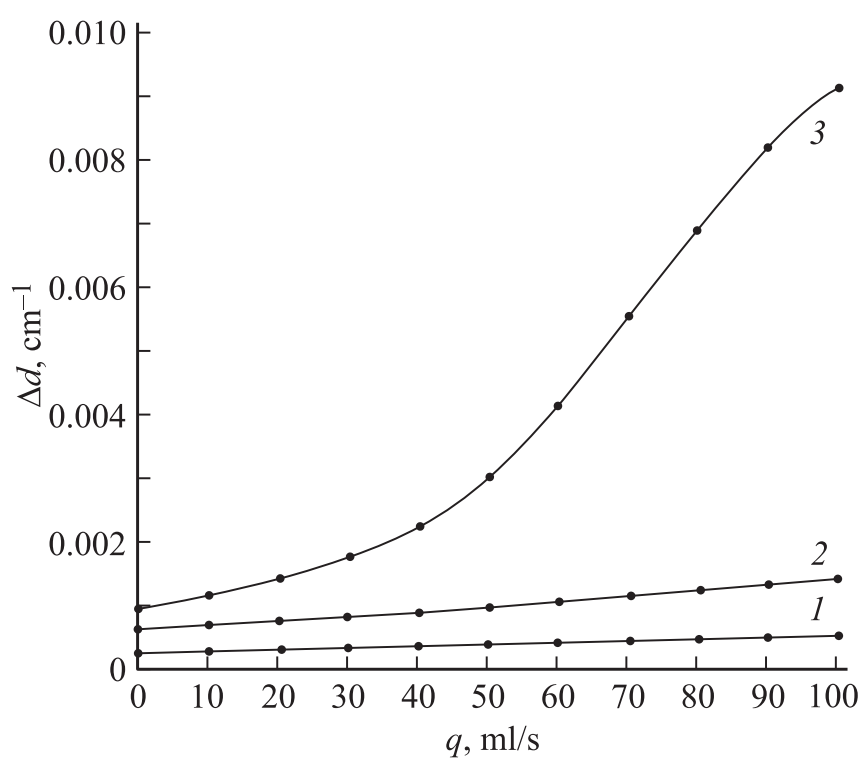

Рис. 4. Зависимость изменения оптической плотности $\Delta d$ от значения расхода $q$ различных сред при $T_{f}=293.2 \mathrm{~K}$. Графики 1,2, 3 соответствуют соответственно воде, водному раствору гидроксида натрия, водному раствору яблочного сока с растворенным сахаром и мякотью.

лученными в работе [26]. Полученные нами экспериментальные результаты подтверждают работоспособность предложенного метода измерения $n_{m}$ текущей среды в разработанном лабораторном макете рефрактометра.

Для различных режимов течения жидкости при стабилизированной температуре в пределах $0.1 \mathrm{~K}$ текущей и стационарной (эталонной) жидких сред было проведено исследование влияние изменения расхода жидкости $q$ на значение $\Delta d$. Далее в соответствии с (3) вычисляется $\delta(\Delta n)$. На рис. 4 в качестве примера представлены зависимости изменения $\Delta d$ от расхода жидкости $q$ в проточной кювете для различных сред.

Проведенные нами исследования показали, что если текущая жидкость однородная (без примесей или добавок, например вода), то независимо от режима ее течения в трубопроводе и диапазоне температур от 283 до $343 \mathrm{~K}$ влияние изменения оптической плотности на погрешность измерения $n_{m}$ несущественно. Данный результат соответствует теоретическим результатам, представленным в работе [25].

Если среда состоит из нескольких компонент (растворенных друг в друге), например водный раствор гидроксида натрия, то по причине неодинакового поглощения света по ширине светового потока в слоях текущей жидкости, особенно при их перемешивании в турбулентном потоке, значение $\Delta d$ незначительно возрастает. Разработанная нами дифференциальная схема регистрации оптического сигнала компенсирует рассогласование в оптических путях лазерных лучей. Это позволяет проводить измерения показателя преломления с погрешностью $10^{-4}$ и меньше. В случае более сложной среды (многофазный раствор), например водный раствор сока с сахаром и мякотью, компенсацию рассогласования оптических путей можно обеспечить только при малых значениях расхода $q$, при которых перемешивания компонент среды незначительные в фиксированном интервале изменения ее температуры. При увеличении $q$ до $30 \mathrm{ml} / \mathrm{s}$ влияние изменения $\Delta d$ на погрешность измерения $\delta(\Delta n)$ существенно увеличивается. Результаты экспериментов позволили нам установить, что для водного раствора яблочного сока с растворенным сахаром и мякотью при значениях $q$ более $40 \mathrm{ml} / \mathrm{s}$ проводить измерения $n_{m}$ с погрешностью $10^{-4}$ и меньше невозможно.

\section{Заключение}

Полученные нами экспериментальные результаты подтвердили возможность исследования влияния оптической плотности текущей жидкости на погрешность измерения показателя преломления $\delta(\Delta n)$ с использованием разработанной нами конструкции лабораторного макета рефрактометра. Проведенные эксперименты показали, что данная конструкция рефрактометра с реализованным в ней для измерения значений $n_{m}$ дифференциальным методом обладает более высокими функциональными возможностями по сравнению ранее используемыми проточными рефрактометрами дифференциального типа.

Установлено, что в однофазных средах с высокой прозрачностью изменение оптической плотности в диапазоне изменения расхода $q$ и температуры $T_{f}$, при которых необходимо осуществлять контроль их состояния, не оказывает существенного влияния на результат измерения показателя преломления $n_{m}$. При контроле состояния многофазных сред (растворов) влияние изменения оптической плотности на результат измерения $n_{m}$ будет зависеть от скорости течения среды 
по трубопроводу (режима течения), а также ее температуры и наличия в ней нерастворенных компонент. Причем для каждой многофазной среды эти факторы необходимо учитывать в индивидуальном порядке. Это позволяет при проведении исследований или контроле состояния текущей среды проводить измерения значений $n_{m}$ с погрешностью $10^{-4}$ и меньше, устанавливая соответствующий расход жидкой среды $q$ с учетом обеспечения необходимого значения ее температуры. В случае отсутствия возможности реализовать необходимое соотношение между этими параметрами $\left(q\right.$ и $\left.T_{f}\right)$, используя полученное нами соотношение (3), можно оценить погрешность измерения $\delta(\Delta n)$. Это позволяет определить целесообразность использования для контроля состояния жидкой среды разработанную нами конструкцию проточного рефрактометра или обосновать использование для измерений $n_{m}$ рефрактометра другого типа с более низкой погрешностью.

\section{Конфликт интересов}

Авторы заявляют, что у них нет конфликта интересов.

\section{Список литературы}

[1] Кашаев Р.С., Газизов Э.Г. // ЖПС. 2010. Т. 77. № 3. С. $347-$ 354.

[2] Давыдов В.В. // Опт. и спектр. 2016. Т. 121. № 1. С. 18-25.

[3] Marusina M.Y., Bazarov B.A., Galaidin P.A., Marusin M.P., Silaev A.A., Zakemovskya E.Y., Mustaev Y.N. // Measurement Techniques. 2014. V. 57. N 5. P. 580-586.

[4] Даев Ж.А. // Измерительная техника. 2016. № 3. С. 29-31.

[5] Nepomnyashchaya E.K., Cheremiskina A.V., Velichko E.N., Aksenov E.T., Bogomaz E.T. // J. Physics: Conference Series. 2015. V. 643(1). P. 012018.

[6] Зубов В.А., Ринкевичюс Б.С. // Квант. электрон. 1997. Т. 24. № 12. C. 1161-1163.

[7] Nepomnyashchaya E.K., Velichko E.N., Aksenov E.T. // J. Physics: Conference Series. 2017. V. 769(4). P. 012025.

[8] Grevtseva A.S., Smirnov K.J., Rud' V.Yu. // J. Physics: Conference Series. 2018. V. 1135(1). P. 012056.

[9] Davydov R.V., Antonov V.I., Yushkova V.V. // J. Phys.: Conf. Ser. 2019. V. 1236(1). P. 012079.

[10] Карабегов М.А. // Измерительная техника. 2004. № 4. C. $50-54$.

[11] Мищенко Ю.В. // Измерительная техника. 1998. № 12. C. $18-22$.

[12] Шур В.Л., Найденов А.С., Лукин А.Я., Лейбенгард Г.И. // Измерительная техника. 2006. № 8. С. 50-53.

[13] Лейбенгард Г.И., Найденов А.С., Шур В.Л. // Измерительная техника. 2004. № 12. С. 58-53.

[14] Мищенко Ю.В. // Измерительная техника. 1995. № 5. C. $20-26$.

[15] Filippov A.V., Rudakova M.A., Skirda V.D. // Magnetic Resonance in Chemistry. 2012. V. 50. N 2. P. 114-119.

[16] Alashkin E.M., Kondratyeva E.T., Kuzmin V.V., Safullin K.R., Stanislavovas A.A., Savinkov A.V., Klochkov A.V., Tagirov M.S. // JEPT Lett. 2018. V. 107. N 2. P. 111-118.
[17] Alexandrov A.S., Ivanov A.A., Archipov R.V., Gafurov M.R., Tagirov M.S. // Magnetic Resonance in Solids. 2019. V. 21. N 2. P. 19203.

[18] Давыдов В.В., Дудкин В.И., Карсеев А.Ю., Вологдин В.А. // ЖПС. 2015. Т. 82. № 6. С. 898-902.

[19] Давыдов В.В. // Измерительная техника. 2016. № 11. C. $46-51$.

[20] Карабегов М.A. // Измерительная техника. 2007. № 6. C. 31-36.

[21] Karabegov M.A. // Measurement Techniques. 2012. V. 55. N 3. P. $478-484$.

[22] Karabegov M.A. // Measurement Techniques. 2012. V. 54. N 11. P. 1203-1212.

[23] Davydov V.V., Kruzhalov S.V., Grebenikova N.M., Smirnov K.J. // Measurement Techniques. 2018. V. 61. N 4. P. 365 372.

[24] Мищенко Ю.В. // Измерительная техника. 2007. № 12. C. $25-30$.

[25] Карабегов М.А., Комарков Ю.И., Хуршудян С.А. // Измерительная техника. 1981. № 3. С. 64-66.

[26] Золотарев В.М., Морозов В.Е., Смирнова Е.В. Оптические постоянные природных и технических сред. СПб.: Изд-во „Лань“, 2008. 348 с. 